\title{
Intestinal microbiota in the pathogenesis of chronic heart failure
}

\author{
Vladimir Ivashkin, ${ }^{1}$ Maria Fadeeva, ${ }^{2}$ Manana Skhirtladze, ${ }^{2}$ Oxana Zolnikova ${ }^{1}$ \\ ${ }^{1}$ Internal Disease Propedeutics; and ${ }^{2}$ Vasilenko Clinic of Internal Disease Propedeutics, Gastroenterology, and Hepatology, \\ I.M. Sechenov First Moscow State Medical University, Moscow, Russian Federation
}

\begin{abstract}
In this review, we have considered and discussed the existing data to achieve a deeper understanding of the role of intestinal microbiota in the development and progression of chronic heart failure (CHF). The key moments of the CHF pathogenesis (an imbalance of neurohumoral systems, inflammatory theory and metabolic disorders) and the respective changes of the intestinal microflora composition were compared.

Here, we also present the latest results of the positive influence of the microflora modulations on the course and prognosis of CHF with the prescribing antibiotics, probiotics and prebiotics.
\end{abstract}

\section{Introduction}

A lot of data were obtained on the role of intestinal microbiota in the pathogenesis of different diseases. ${ }^{1}$ Quantitative and qualitative changes in microbiota have been observed in functional and inflammatory bowel diseases, gastroesophageal reflux disease, liver disease, in kidney, respiratory system, diabetes mellitus, metabolic syndrome, psycho-neurological disorders, etc. In cardiovascular diseases studies it was noticed that the changes in the microbial composition

Correspondence: Oxana Zolnikova, Chair of Internal Disease Propedeutics, I.M. Sechenov First Moscow State Medical University, Moscow, Russian Federation (MSMU), Trubetskaya Str., Bld. 8/2, Moscow 119991, Russia.

Tel.: +79163916056 - Fax: +74992883025.

E-mail:ks.med@mail.ru

Key words: Intestinal microbiota; chronic heart failure; probiotics; cytokines.

Contributions: all authors contributed to this paper with the conception, literature review and analysis; MF, MS, OZ wrote the manuscript; VI reviewed and edited the manuscript critically; all authors approved the final version.

Conflict of interests: the authors declare no potential conflict of interests.

Received for publication: 20 May 2019.

Revision received: 21 July 2019.

Accepted for publication: 27 August 2019.

This work is licensed under a Creative Commons Attribution NonCommercial 4.0 License (CC BY-NC 4.0).

${ }^{\circ}$ Copyright: the Author(s), 2020

Licensee PAGEPress, Italy

Italian Journal of Medicine 2020; 14:1-8

doi:10.4081/itjm.2020.1185 of the intestinal biotope could lead to the development of chronic heart failure. ${ }^{2-4}$ The chronic heart failure (CHF) prevalence varies from 1 to $2 \%$, and reaches $10 \%$ among the elderly in the developed countries. ${ }^{5}$ More than $75 \%$ of patients with CHF over 65 years of age. ${ }^{6}$ The main risk factor for death from CHF is the patient's age, data were obtained by Mazza et al. in the CASTEL study (the Cardiovascular Study in the Elderly). CHF is the most common cause of cardiovascular death for patients over 65 years of age. ${ }^{7}$ Despite modern treatment methods, the prevalence of $\mathrm{CHF}$ and its social significance indicate the need to look for new pathogenically-determined directions in treatment.

At present, CHF is mainly considered from the standpoint of neuroendocrine disorders. It is discussed, that the imbalance of the renin-angiotensin-aldosterone, sympathoadrenal, kinin-kallikrein systems and the system of natriuretic peptides with the development of vasoconstriction and fluid retention, leads to heart remodeling and to a further disruption of its function with the development of metabolic disorders due to the imbalance between the delivery of oxygen and the need for organs and tissues. ${ }^{8}$

However, the increased level of pro-inflammatory cytokines in patients with CHF cannot be explained by the neuroendocrine activation only. According to one of the assumptions, an increase in the level of systemic inflammation factors is due to the lipopolysaccharides of the gram-negative bacteria penetration through the intestinal wall into blood.

\section{The intestinal microbiota changes under chronic heart failure disease}

In the results obtained from some studies a decrease in intestinal microbial diversity in patients with 
CHF was demonstrated. ${ }^{9}{ }^{10}$ Different and sometimes conflicting data for the representation of different taxa were provided in some published articles.

After sequencing 16S-RNA bacteria, those obtained from the fecal samples of the Japanese patients with non-ischemic $\mathrm{CHF}$ and low ejection fraction (CHF-NFV), Katsimichas et al. revealed the differences in $\beta$-diversity for patients with chronic heart failure compared with similar patients without CHF, adjusting the age, the glomerular filtration rate and therapy. Firmicutes and Bacteroidetes dominated in the bacterial community of patients and healthy individuals, simultaneously Proteobacteria were less represented there. In patients with CHF-NFV, Streptococcus and Veillonella were more widely represented and SMB53 were less represented compared with the control group. ${ }^{11}$

The results of some researchers demonstrated a decrease in the number of representatives of Coriobacteriaceae, Erysipelotrichaceae and Ruminococcaceae. ${ }^{9}$ On the other hand, some researches argued in favor of an increase in the number of Streptococcus and Veillonella genera.

In their study, Kummen et al. have shown the changes in the 15 genera of intestinal bacteria in patients with CHF compared with the healthy control group. ${ }^{12}$ It was also detected the increasing number of pathogenic bacteria and fungi, such as Campylobacter, Shigella, Salmonella, Yersinia enterocolitica and Candida under the CHF. ${ }^{13}$

At present, there are no data for the microbial composition of the intestine in patients with CHF with preserved ejection fraction [CHF with preserved ejection fraction $(\mathrm{CHFpEF})]$. The role of the intestinal microbiota has also required clarification in the pathogenesis of chronic heart failure.

However, in according to Wilson Tang et al. there is a relationship between the metabolites been formed in the human body under participating of the intestinal microbiota and the severity of diastolic myocardial dysfunction. So, in particular, the correlation was found between the concentrations of trimethylamine$\mathrm{N}$-oxide, choline, betaine and the value of the $\mathrm{E} / \mathrm{Ea}$ ratio. Nevertheless, it was not noted any significant correlation with the left ventricular (LV) systolic function in this study. ${ }^{14}$

A positive influence of probiotics on an improvement of the diastolic function of myocardium was also found. It resulted in a reducing of the fibrotic changes and the degree of hypertrophy, which confirms the role of intestinal microbiota in the development of cardiac pathology. ${ }^{15}$

At present, it is known and confirmed that the microbiota waste products, short-chain fatty acids (acetate, propionate, butyrate) regulate the metabolic and immune processes of the human body through a sys- tem of the signaling molecules. ${ }^{16}$ An inverse correlation between the number of the butyrate-producing Lachnospiraceae representatives and the T-cell activation marker CD25 (sCD25) was found. The correlation was more pronounced in patients with severe CHF, who had reached the edge point (been included in the waiting list of a heart transplant or dead) during the observation period. In patients who reached the edge point, a decrease in the Eubacterium hallii was also detected, which was one of the main producers of butyrate like the Lachnospiraceae. ${ }^{12}$

Having analyzed the metabolic profile of bacteria in patients with CHF with reduced ejection fraction and healthy individuals, researchers have found differences in the genes that were responsible for the metabolism of amino acids, carbohydrate, vitamins and xenobiotics. ${ }^{11}$

According to the published data, the patients with CHF had age-related differences in the microbiota composition. So, Kamo et al. detected a decrease in Bacteroidetes representatives $\quad(11.7 \pm 2.3 \% \quad v s$ $21.7 \pm 4.1 \%, \mathrm{P}=0.047)$ and a Proteobacteria content increase $(8.4 \pm 2.9 \%$ vs $1.6 \pm 0.5 \%, \mathrm{P}=0.046)$ without significant differences in the Firmicutes for the intestinal microbiota composition in patients with CHF older than 60 years compared with patients before the age of 60 . In accordance with the types of bacteria, in the composition of the intestinal microbiota in feces samples from the elderly patients with CHF, it was found a low content of Faecalibacterium prausnitzii and Clostridium clostridioforme $(3.1 \pm 1.0 \%$ vs $7.5 \pm 1.4 \%$, $\mathrm{P}=0.021, \quad 2.2 \pm 0.6 \% \quad$ vs $6.2 \pm 1.3 \%, \mathrm{P}=0.013$ and $0.7 \pm 0.3 \%$ vs $2.3 \pm 0.6 \%, \mathrm{P}=0.035$, respectively) and a much higher content of Lactobacillus and L. salivarius (21.4 $\pm 5.1 \%$ vs $2.0 \pm 1.1 \%, \mathrm{P}=0.004$ and $14.0 \pm 4.7 \%$ vs $0.4 \pm 0.4 \%, \mathrm{P}=0.018$, respectively) in comparison with the CHF patients from group of younger patients. ${ }^{10}$

\section{The role of microbiota in the inflammatory theory of chronic heart failure}

The cell wall lipopolysaccharides of gram-negative bacteria serve as ligands for Toll-like Type 4 receptors (TLR-4) of the intestinal epithelial cells. In addition, with the reduction of the expression of tight junction proteins in intestinal wall, lipopolysaccharides penetrate into adjacent tissues and bloodstream, where they also interact with type TLR-4 receptors, triggering a cascade of sequential events. This eventually leads to a translocation of the transcription factor NF-kB into the nucleus cells. NF-kB regulates the expression of the genes encoding pro-inflammatory proteins, such as cyclooxygenase- 2 enzyme, nitric oxide synthase, interleukins- $1 \beta, 6$ (IL-1 $\beta$, IL-6), tumor necrosis factor-alpha (TNF- $\alpha$ ). Peptidoglycans of the cell wall interact with nucleotide-binding receptors 
(NOD-receptors). They also activate the immune cells (monocytes, macrophages) and stimulate a release of the regulatory cytokines (Figure 1). Moreover, the peptidoglycan of gram-negative bacteria influences the NOD2 and NOD1 receptors, but the peptidoglycan of gram-positive bacteria influences only the NOD2 receptors. So, the concentration of the bacterial NOD2 agonist reflects a translocation of gram-positive and gram-negative bacteria.

As it was demonstrated in Kim's study on the patients exposed to surgery on the abdominal aorta and the patients with a surgical treatment of the carotid arteries, a concentration of the NOD2 agonist was correlated with the levels of IL-10 and plasma cortisol. In the case of surgery on the abdominal aorta, a positive test for the NOD2 agonist was detected in $90.5 \%$ of the patients studied, while in the second group it was detected just in $23.8 \%$ of the tested patients. The patients belonging to the first group also showed an increased blood concentration of the IL-6, IL-10, Creactive protein, procalcitonin, cortisol. ${ }^{17}$

In the heart failure case, the hemodynamic disturbances lead to hypoxia and edema of the intestinal wall and to the barrier function violation of the intes- tinal wall with its permeability increasing. But from the other side one can lead to a violation of the intestinal motor function, to the syndrome of excessive bacterial growth, to bacterial translocation and to inflammation processes activation.

The most increased concentrations of the endotoxins and pro-inflammatory cytokines in plasma were detected in patients with CHF and edematous-ascitic syndrome. The endotoxins concentration was reduced against the background of the ongoing diuretic therapy. ${ }^{18}$ All this can illustrate the potential contribution of intestinal microbiota and bacterial translocation leading to endotoxemia and immune activation in heart failure pathogenesis.

Sandek et al. studied the transcellular transport and intercellular permeability of the small intestine in 20 patients with CHF [12 patients with peripheral edema and 8 patients without edema with an ejection fraction of $29 \pm 5 \%$, corresponding to New York Heart Association (NYHA) functional class (FC) 2-4]. For this purpose of the study it was used a mixture of sugars was consisted from 3-O-mityl-D-glucose (bound with active transcellular transport with involving of a carrier), D-xylose (a passive transcellular transport by the car-

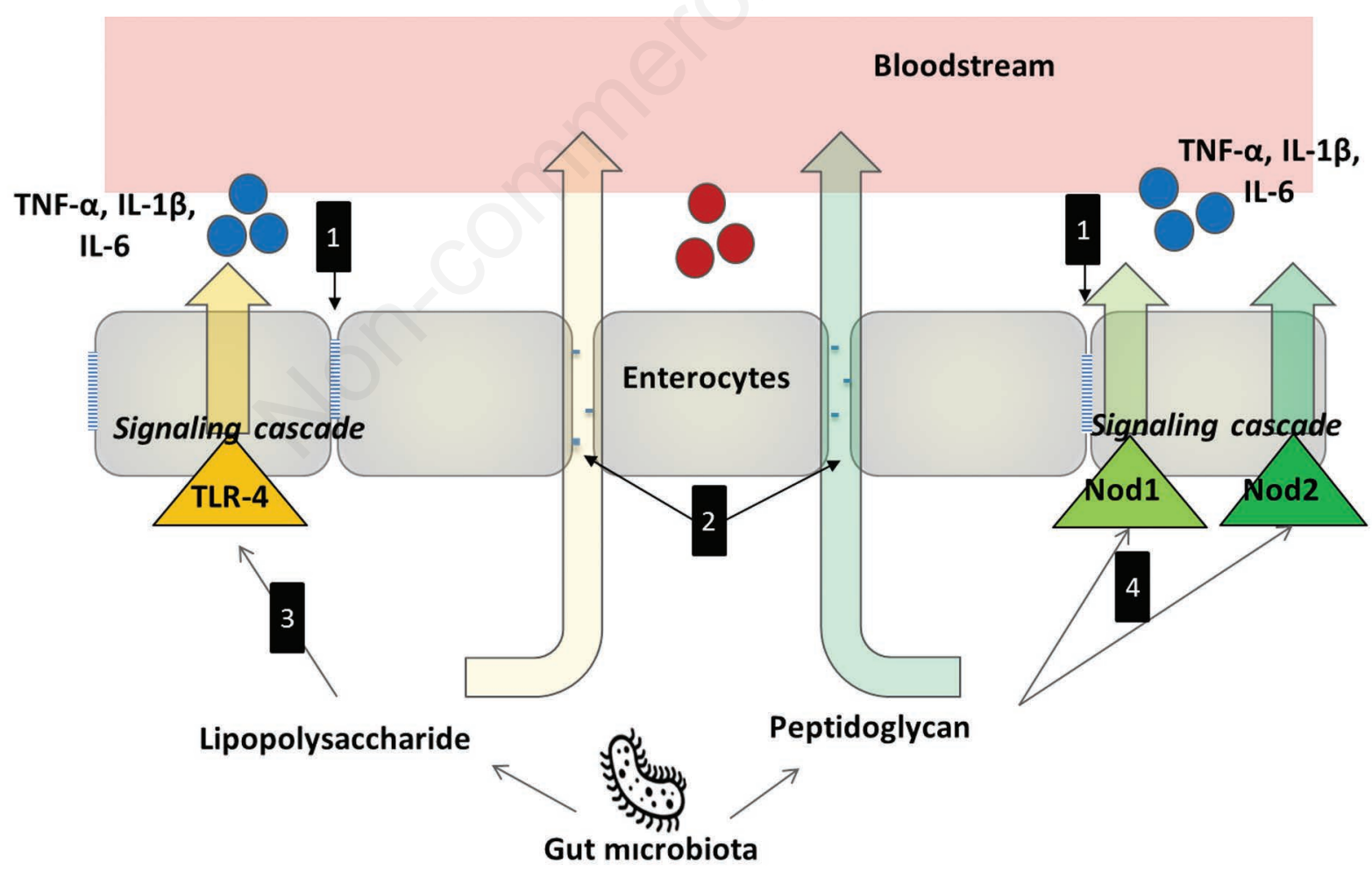

Figure 1. Effects of cell wall components of the gram-positive and gram-negative gut bacteria. 1- The intercellular contacts are normal; 2- a disruption of the intercellular contacts, an increase of intestinal permeability in chronic heart failure, the bacterial translocation; 3- an activation of Toll-like receptors of Type 4 (TLR-4) receptors similar to the lipopolysaccharides kind; 4- an activation of nucleotide-binding receptors (NOD)1- and NOD2-receptors by peptidogly-

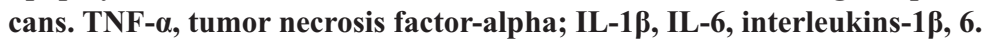


rier), L-ramnosis (diffuse transcellular transport), and melibioses (intercellular transport). An active transcellular transport was slowed down by $54 \%$ in patients with $\mathrm{CHF}$ and these disorders were more pronounced in patients with the edematous syndrome. Also, the study has detected a decrease in the passive vector transport by $34 \%$ in patients with the CHF. That can demonstrate a dysfunction of the intestinal epithelium because of ischemia. The authors had found no significant differences in the allocation of melibiose and Lrhamnose, providing transport without any participation of the carriers in the patients with CHF and from the control group. Furthermore, an increase in concentrations of lipopolysaccharides, TNF and soluble TNF receptors was observed in the patients with edema. A decrease of lipopolysaccharides level was observed after reaching the CHF compensation, showing an existing of a causal link among the gut wall edema, epithelial dysfunction and lipopolysaccharide translocation. ${ }^{19}$

In the earlier work of Koloczek it was detected a violation of the transport of saccharides by carrier proteins and it was not found any correlation with the concentration of circulating cytokines in the patients with peripheral edema. ${ }^{20}$

The permeability of the intestinal wall depends on few factors, including the syndrome of intensive bacterial overgrowth (SIBO) in the small intestine. Lauritano et al. noted that an increase in the intestinal permeability was observed in 11 out of 20 patients with SIBO, while in the control group it was observed in one individual out of 21 patients tested. A decrease in the intestinal wall permeability was achieved after the successful application of rifaximin to decontaminate the wall. ${ }^{21}$

Indirectly, the effect of the SIBO on systemic hemodynamics can be evaluated from the results of studies on patients with cirrhosis of the liver. Decompensated cirrhosis of the liver is characterized by a hyperdynamic type of blood circulation and an increase in cardiac output and circulating blood volume with a decrease in blood pressure and total peripheral vascular resistance. Under the decompensation of cirrhosis of the liver, it is observed an increase in the level of the N-terminal fragment of the precursor of the brain natriuretic peptide (NTproBNP), which is used in the diagnosis and assessment of the severity of heart failure. In patients with the decompensated cirrhosis it was found a direct correlation between the NT-proBNP level and few echocardiographic indicators (edge diastolic volume of the left ventricle, stroke volume, cardiac output, left atrial volume) and an inverse correlation with general peripheral vascular resistance. There was no correlation with the left ventricular ejection fraction. ${ }^{22}$ The role of SIBO has been proven in the development of vasodilation, arterial hypotension and hyperdynamic blood circulation in liver cirrhosis. SIBO was noted more frequently in patients with decompensated liver function and correlated with the severity of disease. ${ }^{23}$

The prevalence of SIBO in patients with CIF was from $38.2 \%$ (according to the results of a hydrogen breath test with lactulose) to $47.1 \%$ (during the methane-based breath test). However, there was no significant correlation among the SIBO presence, inflammatory markers in the blood, NT-proBNP and echocardiography indicators. A positive hydrogen respiratory test was associated with an increased risk of long-term complications (hospitalization, death), while the positive methane breath test did not show a prognostic value. Unfortunately, the results presented in the study were obtained by a small number of participants (patients) and no age-related control group was present. ${ }^{24}$

\section{Inflammation in chronic heart failure}

Pro-inflammatory cytokines play an important role in the pathogenesis of CHF. In particular, it has been established that TNF- $\alpha$ and IL- $1 \beta$ interfere in calcium metabolism in cardiomyocytes and have a negative inotropic effect contributing to the development of cardiac remodeling and reducing the cardiac output. ${ }^{25}$ Along with that, TNF- $\alpha$ and IL-1 $\beta$ are involved in the regulation of Type 1 angiotensin II receptors contributing to the development of myocardial hypertrophy enhancing the processes of fibrosis and apoptosis of cardiomyocytes. ${ }^{26,27}$ So, in the experiment on rats, it was shown that activation of type $1 \mathrm{TNF}-\alpha$ receptor in the brain against the background of the inflammation and increased activity of the renin-angiotensin-aldosterone system leads to an activation of the sympathetic system and results in a progression of $\mathrm{CHF}^{28}$

Besides the hyperactivation of the sympathetic system and the renin-angiotensin-aldosterone system, it is observed an insufficient activity of the natriuretic peptide system during cotransport inhibitory factor. ${ }^{29}$ The atrial natriuretic peptide has an anti-inflammatory and immunomodulating effect due to its influence on the activation of the transcription factor NF-kB, the inflammatory effect of NLRP3, caspase-1 and the interleukin- $1 \beta \mathrm{n}$ release. Thus, a decrease in the activity of natriuretic peptide system contributes to the development of inflammation in $\mathrm{CHF}^{30}$

An increase in the activity of inflammatory mediators (IL-1, 6, 18, TNF- $\alpha, \mathrm{C}$-reactive protein) leads to the progression of the disease in CHF case. It was noted that the levels of pro-inflammatory cytokines were correlated with the severity of CHF and mortality of patients. . $^{31,32}$

So, in our opinion, there is a vicious circle between intestinal microbiota and heart. Chronic heart failure 
causes functional and structural changes in the intestine and the change in the microbial composition contributes to the progression of CHF through the inflammatory cascade.

\section{Trimethylamine-N-oxide in the pathogenesis of chronic heart failure}

Another link of the intestine-heart axis is represented by metabolites arising from the bacteria activity. Trimethylamine-N-oxide (TMAO) is a metabolite of the exogenous choline, phosphatidylcholine (lecithin) and L-carnitine. Jointly with intestinal microbiota they are metabolized to trimethylamine (TMA), which is absorbed into the systemic circulation from the intestine, where it is converted to TMAO. Probably, the TMA metabolism has been provided by the enzymes from the group of flavin monoxidases. In particular, the accumulation of TMA in blood is observed in the presence of a flavin monooxidase- 3 mutation. ${ }^{33}$

The increased TMAO level in plasma is associated with the high cardiovascular risk. ${ }^{34}$ According to data available in literature, TMAO has a pro-atherogenic effect influence on the reverse grip of cholesterol from macrophages. However, the accurate mechanism of that is not completely clear. ${ }^{34,35}$ Nowadays a central role in the pathogenesis of vascular inflammation is defined for the oligomerization of the nucleotide-binding domains (NLRP3) of inflames. Under the TMAO action an activation of the NLRP3-inflammasomes and caspase- 1 is observed. The TMAO contributes to the formation of the reactive forms of oxygen by slowing the activation of manganese-dependent superoxide dismutase- 2 and reducing the expression of sirtuin $3 .{ }^{36}$ The NLRP3-inflammasome and caspase-1 activation is associated with the increased production of IL-1 $\beta$ and the increased endothelial permeability. ${ }^{37}$

In the CHF case was revealed an increase in the level of TMAO. W. Tang. showed that the average concentration of TMAO in the group of the patients with CHF was $5.0 \mu \mathrm{M}$ (IQR 3.0 to $8.5 \mu \mathrm{M}$ ) and that value was significantly higher than in the control group (3.5 $\mu \mathrm{M}$ [IQR 2.3 to $5.7 \mu \mathrm{M}$ ]; $\mathrm{P}<0.001$ ). ${ }^{38}$

In the study on mice, the level of brain natriuretic peptide (the main marker of heart failure) increased with TMAO and choline infusion and it was observed an increase in interstitial and perivascular myocardial fibrosis. ${ }^{39}$

The risk of death among patients with CHF for over 5 years correlates with the level of TMAO [4 quartiles versus 1 : hazard ratio (HR): 3.42 ; $95 \%$ confidence interval (CI): 2.24 to $5.23 ; \mathrm{P}<0.001$, adjusted for traditional cardiovascular risk factors: HR: 1.18; 95\% CI: 1.06 to 1.31 per standard deviation; $\mathrm{P}<0.01$ ], and there were no significant differences between the risk of death in the CHF groups with ischemic and non-ischemic genesis. ${ }^{36}$

The protective properties of TMAO are described in literature. So, spontaneously hypertensive rats were treated with an aqueous solution of TMAO and other rats were treated with placebo (water), no significant differences were detected in blood pressure values, the risk of developing angiopathy and myocardial hypertrophy.

However, against the background of TMAO, during the administration in small doses for a long time (60 weeks) to hypertensive rats, lower values of NTproBNP, vasopressin, left ventricular end-diastolic pressure were observed in comparison with rats treated with placebo (water). Thus, the significant increase of TMAO in plasma did not influence the negative changes in the circulatory system during hypertension, but had a positive effect on the diastolic dysfunction caused by a pressure overload. ${ }^{40}$

\section{Possibilities of intestinal microflora modulations in chronic heart failure}

As it was proved in a number of works, there is a possibility of antibiotics and probiotics exposure on the intestinal microbiota in the CHF cases. In their study, Conraadsa et al. evaluated the effect of selective decontamination of gastrointestinal tract (polymyxin B $800 \mathrm{mg} /$ day, tobramycin $320 \mathrm{mg} /$ day for 8 weeks) on intracellular production cytokine monocytes, circulating cytokine levels (IL-1b, IL-6, TNF-a) and endotoxin, the effect on endothelial function for the 10 patients with the severe CHF form ( 3 and 4 FCs by NYHA, LVEF 14-37\%). The treatment showed a significant decrease in the expression of CD14 monocytes and intracellular production of IL-1b, IL-6 in response to the stimulation with lipopolysaccharide. An endothelial function improvement (increase of flow-mediated dilatation of brachial artery) was noted. However, in the study, no significant changes were detected in the concentration of circulating cytokines and endotoxin. Perhaps, it was due to an insufficient (from the statists point of view) number of observations and an insufficient comparison group (the 8 patients had received placebo). Anti-inflammatory cytokines [for example, the antagonists of the IL-10 and IL-1 (IL$1 \mathrm{Ra})$ receptors] were not evaluated in the study. Therefore, the anti-inflammatory effect of selective decontamination cannot be excluded either. ${ }^{41}$

The positive effects of a number of probiotics in the CHF cases have been noticed in the experimental studies (Table 1). ${ }^{42-45}$ So, the Lactobacillus reuteri GMNL-263 strain resulted in a decrease of the artificially-induced myocardial fibrosis by suppressing the production of transforming growth factor beta (TGF$\beta)$ in the hamsters tested. ${ }^{42}$ In the 8 weeks, a decrease 
in the left ventricular ejection fraction against the background of increased nutrition was observed in the hamsters tested. An increase of the ejection fraction was observed in the case of the probiotic treatment with Lactobacillus reuteri GMNL-263. Against the background of a high-fat diet, cardiomyocytes were located randomly and the intercellular distance was increased too. These changes were not too clearly pronounced, the cardiomyocyte apoptosis was slowed down in the probiotic group. ${ }^{43}$

In the study on rats with bandaged coronary artery, the Lactobacillus rhamnosus GR1 strain reduced myocardial mass and the level of atrial natriuretic peptide. There was also an improvement in the systolic and diastolic functions of the heart in rats from group GR-1. ${ }^{44}$

On a background of a high-fat diet using the multistrain probiotic containing Lactobacillus rhamnosus, Pediococcus acidilactici, Bifidobacterium adolescentis, a less pronounced change was noticed in the structure of rats myocardium.

Also it was noted a slowdown in the development of fibrosis and myocardial hypertrophy, which was revealed due to the suppression of the TGF/MMP2/MMP9 profibrotic pathway (transforming growth factor/matrix metalloproteinase-2/matrix metalloproteinase-9) and the ERK5/uPA/ANP pathway (regulated kinase-5/urokinase-type plasminogen activator/atrial natriuretic peptide) in the rats studygroup treated by probiotic. ${ }^{44}$

With the use of probiotic bacteria, encouraging results of an improvement in the systolic function of the left ventricle in patients with CHF were demonstrated in some clinical studies. Thus, a decrease in the size of the left atrium and increase in the ejection fraction were detected among patients with CHF NYHA II or III FC with LV below $50 \%$ in the group been treated by the probiotic with Saccharomyces boulardii. ${ }^{45}$

There are not enough published studies of the effects of prebiotics in CHF yet. The importance of dietary fiber deficiency in patients with $\mathrm{CHF}$ and the anti-inflammatory properties of prebiotic doses of lactulose have been observed. ${ }^{46,47}$

One of the promising research directions is the probiotics influence on the metabolic pathways through which the gut microbes can effect on the host organism. For example, the TMAO production decrease can be achieved by blocking the enzyme choline-TMA-lyase, which converts choline to TMA. ${ }^{48}$ Among the choline-TMA-lyase inhibitors, there is 3,3-dimethyl-1-butanol, that is contained in cold-pressed olive oil. ${ }^{49}$ This could determine the positive results of the Mediterranean diet for those patients suffering from CHF, by reducing TMAO and improving their prognosis. ${ }^{50}$

\section{Conclusions}

The existing data on intestinal microbiota composition in patients with chronic heart failure are contradictory, nevertheless, the effect of intestinal bacteria has been defined in the development of this disease. Microbial metabolites and metabolites were formed by an action of intestinal microflora, these metabolites influence the CHF course and correlate with the disease severity.

In particular, by enhancing the production of inflammatory cytokines, the trimethylamine-N-oxide contributes to the endothelium permeability increase with the development of angiopathy, accelerates the processes of myocardial remodeling, and increases the fibrosis. Bacterial translocation also leads to the increasing inflammation in the CHF case.

Predominantly, the role of intestinal microbiota in the pathogenesis of CHFpEF can be explained by the bacterial translocation against the background of the intestinal wall permeability increase and the inflammatory response been mediated by some cytokines with the negative inotropic effect.

SIBO plays an important role by increasing the bacterial translocation through the intestinal wall. Moreover, in CHF cases, SIBO is associated with the high

Table 1. Effects of probiotics in chronic heart failure.

\begin{tabular}{|c|c|}
\hline Bacteria strains & Results \\
\hline L. reuteri GMNL-263 & Reduction of myocardial fibrosis by suppressing with transforming growth factor $\beta^{42}$ \\
\hline L. reuteri GMNL-263 & $\begin{array}{l}\text { A fraction increase in left ventricular ejection, slowing down of the myocardial remodeling and cardiomyocyte apoptosis } \\
\text { processes }^{42}\end{array}$ \\
\hline L. rhamnosus $G R-1$ & $\begin{array}{l}\text { Reduction of myocardial volume and atrial natriuretic peptide level, an improvement of systolic and diastolic functions } \\
\text { during coronary artery ligation }{ }^{43}\end{array}$ \\
\hline $\begin{array}{l}\text { L. rhamnosus, } \\
\text { P. acidilactici, } \\
\text { B. adolescentis }\end{array}$ & A slowing down of the fibrosis and myocardial hypertrophy processes ${ }^{44}$ \\
\hline S. boulardii & The reduction of left atrium size and the increase of the left ventricle ejection fraction ${ }^{45}$ \\
\hline
\end{tabular}


risk of the repeated hospitalizations and patients' death.

The microbiota effect on CHFpEF requires a clarification. There are some indications concerning the microbiota influence mediated by metabolites on myocardial hypertrophy, fibrosis and diastolic function of the myocardium, which can be considered as the possible CHFpEF mechanisms:

- the common matter of the CHF pathogenesis (imbalance of neurohumoral systems, inflammatory theory and metabolic disorders) and the changes in violation of the composition of intestinal microbiota;

- the positive effects of probiotics on the microbial composition of intestine and on the course and prognosis in CHF cases;

- an opportunity to influence on the metabolic pathways of the microbe - heart communication.

All of these could make possible to consider the microbiome as the marker and possible target in the treatment of the CHF disease. Certainly, further researches are needed in this area.

\section{References}

1. Sekirov I, Russell SL, Antunes LC, Finlay BB. Gut microbiota in health and disease. Physiol Rev 2010;90: 859-904.

2. Ettinger G, MacDonald K, Reid G, Burton JP. The influence of the human microbiome and probiotics on cardiovascular health. Gut Microb 2014;5:719-28.

3. Yang T, Santisteban MM, Rodriguez V, et al. Gut microbiota dysbiosis is linked to hypertension. Hypertension 2015;65:1331-40.

4. Karbach SH, Schönfelder T, Brandão I, et al. Gut microbiota promote angiotensin II-induced arterial hypertension and vascular dysfunction. J Am Heart Assoc 2016;30:5-9.

5. Mosterd A, Hoes AW. Clinical epidemiology of heart failure. Heart 2007;93:1137-46.

6. US Department of Health and Human Services. Morbidity and mortality: 1998 chartbook on cardiovascular, lung, and blood diseases. Bethesda, MD: US Department of Health and Human Services; 1998. Available from: www.nhlbi.nih.gov/nhlbi/seiin//other/cht-book/htm

7. Mazza A, Tikhonoff V, Casiglia E, Pessina AC. Predictors of congestive heart failure mortality in elderly people from the general population the Cardiovascular Study in the ELderly (CASTEL). Int Heart J 2005;46:419-31.

8. Fomin IV. Chronic heart failure in Russian Federation: what do we know and what to do. Russian Journal of Cardiology. 2016;(8):7-13.(In Russ.)

9. Luedde M., Winkler Th., Heinsen F. et al. Heart failure is associated with depletion of core intestinal microbiota. ESC Heart Failure 2017;4:282-90.

10. Kamo T, Akazawa H, Suda W, et al. Dysbiosis and compositional alterations with aging in the gut microbiota of patients with heart failure. PLoS One 2017;12:e174099.

11. Katsimichas T, Ohtani T, Motooka D, et al. Non-is- chemic heart failure with reduced ejection fraction is associated with altered intestinal microbiota. Circ J 2018;82:1640-50.

12. Kummen M, Mayerhofer CCK, Vestad B, et al. Gut microbiota signature in heart failure defined from profiling of 2 independent cohorts. J Am Coll Cardiol 2018;71:1184-86.

13. Pasini E, Aquilani R, Testa C, et al. Pathogenic gut flora in patients with chronic heart failure. J Am Coll Cardiol HF 2016;4:220-7.

14. Tang WHW, Li DY, Hazen SL. Dietary metabolism, the gut microbiome, and heart failure. Nat Rev Cardiol 2019;16:137-54.

15. Gan XT, Ettinger G, Huang CX, et al. Probiotic administration attenuates myocardial hypertrophy and heart failure after myocardial infarction in the rat. Circ Heart Fail 2014;7:491-9.

16. Arpaia N, Campbell C, Fan X, et al. Metabolites produced by commensal bacteria promote peripheral regulatory T-cell generation. Nature 2013;504:451-5.

17. Kim OY, Monsel A, Bertrand MS, et al. Translocation of bacterial NOD2 agonist and its link with inflammation. Critical Care 2009; R124.

18. Niebauer J, Volk H, Kemp M, et al. Endotoxin and immune activation in chronic heart failure: a prospective cohort study. Lancet 1999;353:1838-42.

19. Sandik A, Bjarnason I, Volk HD, et al. Studies on bacterial endotoxin and intestinal absorption function in patients with chronic heart failure. Int $\mathrm{J}$ Cardiol 2012;157:80-5.

20. Koloczek V, Rauchhaus M, Kemp M, et al. Markers of intestinal ischaemia relate to proinflammatory cytokines in patients with congestive heart failure. Eur Heart $\mathrm{J}$ 2000;21:662.

21. Lauritano EC, Valenza V, Sparano L, et al. Small intestinal bacterial overgrowth and intestinal permeability. Scand J Gastroenterol 2010;45:1131-2.

22. Maslennikov R, Driga A, Ivashkin K, Ivashkin V. NTproBNP as a biomarker for hyperdynamic circulation in decompensated cirrhosis. Gastroenterol Hepatol Bed Bench 2018;11:325-32.

23. Maslennikov RV, Driga AA, Ivashkin KV, et al. Small intestinal bacterial overgrowth syndrome and systemic inflammation in pathogenesis of hemodynamic changes at liver cirrhosis. Ross Z Gastroenterol Gepatol Koloproktol 2017;27:45-56.

24. Mollar A, Villanueva MP, Núñez E, et al. Hydrogen- and methane-based breath testing and outcomes in patients with heart failure. J Card Fail 2019;pii:S10719164(18)31106-0.

25. Yokoyama T, Vaca L, Rossen RD, et al. Cellular basis for the negative inotropic effects of tumor necrosis factor-alpha in the adult mammalian heart. J Clin Invest 1993;92:2303-12.

26. Gurantz D, Cowling RT, Varki N, et al. IL-1beta and TNF-alpha upregulate angiotensin II type 1 (AT1) receptors on cardiac fibroblasts and are associated with increased AT1 density in the post-MI heart. J Mol Cell Cardiol 2005;38:505-15.

27. Chandrasekar B, Vemula K, Surabhi RM, et al. Activation of intrinsic and extrinsic proapoptotic signaling pathways in interleukin-18-mediated human cardiac endothelial cell death. J Biol Chem 2004;279:20221-33. 
28. Yu Y, Wei SG, Weiss RM, Felder RB. TNF- $\alpha$ receptor 1 knockdown in the subfornical organ ameliorates sympathetic excitation and cardiac hemodynamics in heart failure rats. Am J Physiol Heart Circ Physiol 2017;313:744-56.

29. Schrier RW, Abraham WT. Hormones and hemodynamics in heart failure. N Engl J Med 1999;341:577-85.

30. Mezzasoma L, Antognelli C, Talesa VN. Atrial natriuretic peptide down-regulates LPS/ATP-mediated IL-1 $\beta$ release by inhibiting NF-kB, NLRP3 inflammasome and caspase-1 activation in THP-1 cells. Immunol Res 2016;64:303-12.

31. Bozkurt B, Mann DL, Deswal A. Biomarkers of inflammation in heart failure. Heart Fail Rev 2010;15:331-41.

32. Rauchhaus M, Doehner W, Francis DP, et al. Plasma cytokine parameters and mortality in patients with chronic heart failure. Circulation 2000;102:3060-7.

33. Treacy EP, Akerman BR, Chow LM, et al. Mutations of the flavin-containing monooxygenase gene (FMO3) cause trimethylaminuria, a defect in detoxication. Hum Mol Genet 1998;7:839-45.

34. Tang WH, Wang Z, Levison BS, et al. Intestinal microbial metabolism of phosphatidylcholine and cardiovascular risk. N Engl J Med 2013;368:1575-84.

35. Bennett BJ, de Aguiar Vallim TQ, Wang Z, et al. Trimethylamine-N-oxide, a metabolite associated with atherosclerosis, exhibits complex genetic and dietary regulation. Cell Metab 2013;17:49-60.

36. Chen ML, Zhu XH, Ran L, et al. Trimethylamine-Noxide induces vascular inflammation by activating the NLRP3 inflammasome through the SIRT3-SOD2mtROS signaling pathway. J Am Heart Assoc 2017;6:e006347.

37. Boini KM, Hussain T, Li PL, Koka S. Trimethylamine$\mathrm{N}$-oxide instigates NLRP3 inflammasome activation and endothelial dysfunction. Cell Physiol Biochem 2017;44:152-62.

38. Tang WH, Wang Z, Fan Y, et al. Prognostic value of elevated levels of intestinal microbe-generated metabolite trimethylamine-N-oxide in patients with heart failure: refining the gut hypothesis. Am Coll Cardiol 2014;64:1908-14.

39. Organ CL, Otsuka H, Bhushan S, et al. Choline diet and its gut microbe-derived metabolite, trimethylamine N- oxide, exacerbate pressure overload-induced heart failure. Circ Heart Fail 2016;9:e002314.

40. Huc T, Drapala A, Gawrys M, et al. Chronic, low-dose TMAO treatment reduces diastolic dysfunction and heart fibrosis in hypertensive rats. Am J Physiol Heart Circ Physiol 2018;315:H1805-20

41. Conraads VM, Jorens PG, De Clerck LS, et al. Selective intestinal decontamination in advanced chronic heart failure: a pilot trial. Eur J Heart Fail 2004;6:483-91.

42. Ting WJ, Kuo WW, Hsieh DJ, et al. Heat killed Lactobacillus reuteri GMNL-263 reduces fibrosis effects on the liver and heart in high fat diet-hamsters via TGF- $\beta$ suppression. Int J Mol Sci 2015;16:25881-96.

43. Ting WJ, Kuo WW, Kuo CH, et al. Supplementary heatkilled Lactobacillus reuteri GMNL-263 a meliorates hyperlipidaemic and cardiac apoptosis in high-fat diet-fed hamsters to maintain cardiovascular function. Br J Nutr 2015;114:706-12.

44. Lai CH, Tsai CC, Kuo WW, et al. Multi-strain probiotics inhibit cardiac myopathies and autophagy to prevent heart injury in high-fat diet-fed rats. Int J Med Sci 2016;13:277-85.

45. Costanza AC, Moscavitch SD, Faria Neto HCC, Mesquita ET. Probiotic therapy with Saccharomyces boulardii for heart failure patients: A randomized, double-blind, placebo-controlled pilot trial. Int J Cardiol 2015;179:348-50.

46. Makarewicz-Wujec M, Parol G, Parzonko A, Kozłowska-Wojciechowska M. Supplementation with omega-3 acids after myocardial infarction and modification of inflammatory markers in light of the patients' diet: a preliminary study. Kardiol Pol 2017;75:674-81.

47. Singh RK, Chang HW, Yan D, et al. Influence of diet on the gut microbiome and implications for human health. J Transl Med 2017; 15:73.

48. Craciun S, Balskus EP. Microbial conversion of choline to trimethylamine requires a glycyl radical enzyme. Proc Natl Acad Sci U S A 2012;109:21307-12.

49. Wang Z. Non-lethal inhibition of gut microbial trimethylamine production for the treatment of atherosclerosis. Cell 2015;163:1585-95.

50. Papadaki A. Mediterranean diet and risk of heart failure: results from the PREDIMED randomized controlled trial. Eur J Heart Fail 2017;19:1179-85. 